\title{
Extensions of classical hypergeometric identities of Bailey and Whipple
}

\author{
Ilia D. Mishev * \\ Dedicated to the memory of Professor Plamen Djakov.
}

\begin{abstract}
We obtain extensions of classical hypergeometric identities of Bailey and Whipple that transform nearly-poised and very-well-poised series to Saalschützian series, Saalschützian series to Saalschützian series, and very-well-poised and nearly-poised series to very-well-poised series. We employ a method in which summations and transformations of lower-order series are used to obtain transformations of higher-order series. By taking limits, we also obtain extensions of two classical quadratic transformations of Whipple and Bailey. Furthermore, we show how a number of other well-known results regarding hypergeometric series follow as special cases of our results.
\end{abstract}

\section{Introduction}

Identities among hypergeometric series, both terminating and nonterminating, have been the subject of extensive research. In the 1920s, Bailey and Whipple (see [1, 18, 19, 20, 21, 22]) found a number of identities relating various terminating hypergeometric series that are listed in Chapters 4 and 7 of Bailey's tract [2]. In this paper, we generalize these terminating hypergeometric identities found by Bailey and Whipple and extend them to higher-order hypergeometric series.

\footnotetext{
*Department of Mathematics, University of Colorado Boulder, Campus Box 395, Boulder, CO 80309-0395, U.S.A. E-mail address: ilia.mishev@colorado.edu
} 
We consider classical identities involving terminating very-well-poised, nearly-poised and Saalschützian series (see Section 2 for the relevant definitions). The highest order classical transformation formula between two terminating very-well-poised series was found by Bailey in [1] and involves two terminating very-well-poised ${ }_{9} F_{8}(1)$ series (see [2, Eq. 4.3.7]). This formula was recently extended to the ${ }_{11} F_{10}$ level by Srivastava, Vyas and Fatawat $[15$, Theorem 3.4] by adding two new numerator and denominator pairs of parameters that differ by one each. In this paper, we further extend Srivastava, Vyas and Fatawat's result to a relation involving two terminating very-wellpoised ${ }_{13} F_{12}(1)$ series (see Proposition 5.3 in Section 5) by adding two more pairs of numerator and denominator parameters with unit difference.

In Section 4.5 of Bailey's tract [2], one can find a number of transformation formulas involving terminating nearly-poised series discovered by Whipple and Bailey. The identities [2, Eq. 4.5.1] (originally found by Whipple in [22]) and [2, Eq. 4.5.2] (originally found by Bailey in [1]) relate nearly-poised ${ }_{4} F_{3}(1)$ and ${ }_{5} F_{4}(1)$ series, respectively, to Saalschützian ${ }_{5} F_{4}(1)$ series. In this paper (see Corollary 3.5 in Section 3), we extend these two identities to a single transformation formula between a ${ }_{5} F_{4}(1)$ series, which is not nearly-poised but in which two pairs of numerator and denominator parameters deviate from "well-poisedness", and a Saalschützian ${ }_{6} F_{5}(1)$ series. In addition, this extension is further generalized by Proposition 3.3 in Section 3 to a relation between two terminating ${ }_{7} F_{6}(1)$ series, one of which is Saalschützian. A special case of Proposition 3.3 given in Corollary 3.4 coincides with a recent special case of a result of Maier [9].

The classical Whipple transformation between a very-well-poised ${ }_{7} F_{6}(1)$ and a Saalschützian ${ }_{4} F_{3}(1)$ series (see [19], 21] and [2, Eq. 4.3.4]) was recently generalized by Srivastava, Vyas and Fatawat [15, Theorem 3.2]. In this paper, we provide a very general result in Proposition 3.6 that extends both Srivastava, Vyas and Fatawat's result [15, Theorem 3.2] and the results described in the paragraph above.

In Section 4 of this paper, we study transformations between two terminating Saalschützian series. The classical result in this area is the Whipple transform (see [19], 20] and [2, Eq. 7.2.1]) involving two terminating Saalschützian ${ }_{4} F_{3}(1)$ series. In Proposition 4.2 in Section 4, we extend the Whipple transform to a transformation that involves two terminating Saalschützian ${ }_{6} F_{5}(1)$ series in each of which series two numerator parameters exceed two denominator parameters by one.

In Section 5, in addition to obtaining the above-mentioned relation in- 
volving two terminating very-well-poised ${ }_{13} F_{12}(1)$ series, we also obtain extensions of classical results found by Bailey in [1, Eqs. 8.1, 8.2 and 8.3] and reproduced in [2, Eqs. 4.5.3, 4.5.4 and 4.5.5] that transform terminating nearly-poised ${ }_{5} F_{4}(1)$ series with parametric excesses $\omega=1$ and $\omega=2$ and a terminating nearly-poised ${ }_{6} F_{5}(1)$ series with parametric excess $\omega=1$ to terminating very-well-poised ${ }_{9} F_{8}(1)$ series. The immediate extension of $[2$, Eqs. 4.5.3, 4.5.4 and 4.5.5] is given in Corollary [5.5, and a further extension is provided in Proposition 5.4.

To obtain our results, we use a method employed by Bailey in [1] and [2, Chapter 4] that utilizes sums of series of lower order to obtain transformations of series of higher order. The extensions of Bailey's general formulas [2, Eqs. 4.3.1 and 4.3.6] are provided in Propositions 3.1 and 5.1, respectively. We should point out that Bailey uses the Pfaff-Saalschütz formula (see [2, Eq. 2.2.1]) to obtain [2, Eq. 4.3.1] while we use the extension of the PfaffSaalschütz formula to a ${ }_{4} F_{3}(1)$ series given by Rakha and Rathie [12] to obtain the more general Proposition 3.1. Moreover, Bailey uses Dougall's theorem (see [5, Eq. 6], [7] and [2, Eq. 4.3.5]) to obtain [2, Eq. 4.3.6] and we use the extension of Dougall's theorem to a ${ }_{9} F_{8}(1)$ series summation provided by Srivastava, Vyas and Fatawat [15, Theorem 3.3] to prove the more general Proposition 5.1. Finally, in this paper, not only do we use the method just described with known summations of series, but we also use it with a known transformation of series and then reverse the order of summation to obtain a new transformation (see Proposition 3.6).

The method described above that we use in this paper is parallel to the Bailey's transform (see [3], 4] and [14, pp. 58-74]), which is employed by Srivastava, Vyas and Fatawat in [15]. Both methods can be utilized to obtain many of the known transformations of hypergeometric series. We should also note that, according to the Karlsson-Minton summation formula (see [8]), any hypergeometric series in which numerator and denominator parameters differ by positive integers can be written as a finite sum of hypergeometric series of lower order, but we have not used this approach or formula in our paper.

Finally, in Section 6, by taking certain limits of relations in Section 3, we obtain extensions of some classical quadratic transformations of hypergeometric series. In particular, we obtain extensions in terms of single transformations of both the quadratic transformation found by Whipple in [22, Eq. (7.1)] and its companion transformation found by Bailey in [1, Eq. (9.1)]. Also, a special case of our most general extension there (see (6.3) in Section 6 
below) coincides with a special case of a transformation of Maier [9, Theorem 3.4 .

\section{Preliminaries}

The hypergeometric series of type ${ }_{r} F_{s}$ is defined by

$$
{ }_{r} F_{s}\left[\begin{array}{l}
a_{1}, a_{2}, \ldots, a_{r} ; \\
b_{1}, b_{2}, \ldots, b_{s} ;
\end{array}\right]=\sum_{n=0}^{\infty} \frac{\left(a_{1}\right)_{n}\left(a_{2}\right)_{n} \cdots\left(a_{r}\right)_{n}}{n !\left(b_{1}\right)_{n}\left(b_{2}\right)_{n} \cdots\left(b_{s}\right)_{n}} z^{n}
$$

where $r$ and $s$ are nonnegative integers, $a_{1}, a_{2}, \ldots, a_{r}, b_{1}, b_{2}, \ldots, b_{s}, z \in \mathbb{C}$, and the rising factorial $(a)_{n}$ is given by

$$
(a)_{n}= \begin{cases}a(a+1) \cdots(a+n-1), & n>0 \\ 1, & n=0 .\end{cases}
$$

In this paper we will be mostly interested in the case where $r=s+1$. The series of type ${ }_{s+1} F_{s}$ converges absolutely if $|z|<1$ or if $|z|=1$ and $\operatorname{Re}\left(\sum_{i=1}^{s} b_{i}-\sum_{i=1}^{s+1} a_{i}\right)>0$ (see [2, p. 8]). We assume that no denominator parameter $b_{1}, b_{2}, \ldots, b_{s}$ is a negative integer or zero. If a numerator parameter $a_{1}, a_{2}, \ldots, a_{s+1}$ is a negative integer or zero, the series has only finitely many nonzero terms and is said to terminate.

When $z=1$, we say that the series is of unit argument and of type ${ }_{s+1} F_{s}(1)$. If $\sum_{i=1}^{s} b_{i}-\sum_{i=1}^{s+1} a_{i}=1$, the series is called Saalschützian. If $1+a_{1}=b_{1}+a_{2}=\cdots=b_{s}+a_{s+1}$, the series is called well-poised. A well-poised series that satisfies $a_{2}=1+\frac{1}{2} a_{1}$ is called very-well-poised. The parametric excess $\omega$ is given by $\omega=\sum_{i=1}^{s} b_{i}-\sum_{i=1}^{s+1} a_{i}$. Note that $\omega=1$ for a Saalschützian series.

We will use the following extension of the classical $\mathrm{Chu}$-Vandermonde formula (see [2, Section 1.3]), which extension sums a special terminating ${ }_{3} F_{2}(1)$ series where a numerator parameter exceeds a denominator parameter by one:

$$
{ }_{3} F_{2}\left(\begin{array}{c|c}
a, p+1,-n & 1 \\
b, p & 1
\end{array}\right)=\frac{(b-a-1)_{n}(q+1)_{n}}{(b)_{n}(q)_{n}},
$$

where

$$
q=\frac{p(b-a-1)}{p-a}
$$


The above formula (2.2) appears in [10]. A nonterminating version of the same formula can be found in [11, p. 534, Eq. (10)]. Letting $p \rightarrow \infty$ in (2.2) yields the $\mathrm{Chu}$-Vandermonde formula.

We will also use the following extension of the Pfaff-Saalschütz formula (see [2, Eq. 2.2.1]) given by Rakha and Rathie [12] which finds the sum of a special terminating Saalschützian ${ }_{4} F_{3}(1)$ series where a numerator parameter exceeds a denominator parameter by one:

$$
\begin{aligned}
& { }_{4} F_{3}\left(\begin{array}{c}
a, b, p+1,-n \\
c, p, 2+a+b-c-n
\end{array} \mid \begin{array}{l}
1
\end{array}\right) \\
& =\frac{(c-a-1)_{n}(c-b-1)_{n}(q+1)_{n}}{(c)_{n}(c-a-b-1)_{n}(q)_{n}},
\end{aligned}
$$

where

$$
q=\frac{p(c-a-1)(c-b-1)}{a b+p(c-a-b-1)} .
$$

Letting $p=b$ in (2.4) yields the Pfaff-Saalschütz formula, while letting $b \rightarrow \infty$ in (2.4) gives (2.2) .

We note that (2.4) can also be written as

$$
\begin{aligned}
& { }_{4} F_{3}\left(\begin{array}{c}
a-b-c, \gamma_{1}+1, a+n,-n \\
1+a-b, 1+a-c, \gamma_{1}
\end{array} \mid 1\right) \\
& =\frac{(b)_{n}(c)_{n}(a-p+1)_{n}(p+1)_{n}}{(1+a-b)_{n}(1+a-c)_{n}(p)_{n}(a-p)_{n}},
\end{aligned}
$$

where

$$
\gamma_{1}=\frac{p(a-p)(b+c-a)}{b c-p(a-p)}
$$

and as

$$
\begin{aligned}
& { }_{4} F_{3}\left(\begin{array}{c}
c-a-1, c-b-1, \gamma_{2}+1,-n \\
c, \gamma_{2}, c-a-b-n
\end{array} \mid 1\right) \\
& =\frac{(a)_{n}(b)_{n}(p+1)_{n}}{(c)_{n}(1+a+b-c)_{n}(p)_{n}}
\end{aligned}
$$

where

$$
\gamma_{2}=\frac{p(c-a-1)(c-b-1)}{a b+p(c-a-b-1)} .
$$


We will use (2.6) and (2.8) in Sections 3 and 4, respectively, where we study extensions of transformations of nearly-poised and very-well-poised series to Saalschützian series and extensions of transformations of Saalschützian series to Saalschützian series.

In [15, Theorem 3.3], Srivastava, Vyas and Fatawat find a generalization of the classical Dougall's theorem for the sum of a terminating very-wellpoised ${ }_{7} F_{6}(1)$ series with parametric excess $\omega=2$ (see [5, Eq. 6], [7] and [2, Eq. 4.3.5]). The generalization found by Srivastava, Vyas and Fatawat can be written as

$$
\begin{aligned}
& { }_{9} F_{8}\left(\begin{array}{c}
a, 1+\frac{a}{2}, b, c, d, \\
\frac{a}{2}, 1+a-b, 1+a-c, 1+a-d,
\end{array}\right. \\
& \left.\begin{array}{c}
2 a-b-c-d+n, a-p+1, p+1,-n ; \mid \\
1+b+c+d-a-n, p, a-p, 1+a+n ; \mid
\end{array}\right) \\
& =\frac{(1+a)_{n}(a-b-c)_{n}(a-b-d)_{n}(a-c-d)_{n}(\alpha+1)_{n}}{(1+a-b)_{n}(1+a-c)_{n}(1+a-d)_{n}(a-b-c-d)_{n}(\alpha)_{n}},
\end{aligned}
$$

where

$$
\alpha=\frac{p(a-p)(a-b-c)(a-b-d)(a-c-d)}{(2 a-b-c-d+n)(b c d+p(a-p)(a-b-c-d))} .
$$

Dougall's theorem follows from (2.10) by letting $p=b$. We note that (2.10) can also be written as

$$
\begin{aligned}
& { }_{9} F_{8}\left(\begin{array}{c}
\lambda, 1+\frac{\lambda}{2}, \lambda+b-a, \lambda+c-a, \lambda+d-a, \\
\frac{\lambda}{2}, 1+a-b, 1+a-c, 1+a-d,
\end{array}\right. \\
& \left.\begin{array}{c|c}
a+n, \frac{\lambda}{2}-\gamma+1, \frac{\lambda}{2}+\gamma+1,-n ; & 1 \\
+\lambda-a-n, \frac{\lambda}{2}+\gamma, \frac{\lambda}{2}-\gamma, 1+\lambda+n ; & 1
\end{array}\right) \\
& =\frac{(1+\lambda)_{n}(b)_{n}(c)_{n}(d)_{n}(a-p+1)_{n}(p+1)_{n}}{(a-\lambda)_{n}(1+a-b)_{n}(1+a-c)_{n}(1+a-d)_{n}(p)_{n}(a-p)_{n}},
\end{aligned}
$$

where

$$
\lambda=2 a-b-c-d
$$

and

$$
\gamma^{2}=\frac{\lambda^{2}}{4}-\frac{p(a-p)(a-b-c)(a-b-d)(a-c-d)}{b c d+p(a-p)(a-b-c-d)} .
$$

We will use (2.12) in Section 5 where we study extensions of transformations of very-well-poised and nearly-poised series to very-well-poised series. 


\section{Extensions of hypergeometric transforma- tions of nearly-poised and very-well-poised series to Saalschützian series}

In this section, we study extensions of the classical identities given in [2, Eqs. 4.5.1, 4.5.2 and 4.3.4]. We begin with a general formula that extends [2, Eq. 4.3.1]:

Proposition 3.1. Let

$$
\gamma=\frac{p(a-p)(b+c-a)}{b c-p(a-p)}
$$

Then

$$
\begin{aligned}
& { }_{r+6} F_{s+4}\left(\begin{array}{c}
a, b, c, a-p+1, p+1, a_{1}, \ldots, a_{r},-n \\
1+a-b, 1+a-c, p, a-p, b_{1}, \ldots, b_{s}
\end{array} \mid x\right) \\
& =\sum_{m=0}^{n}\left(\frac{\left(\frac{a}{2}\right)_{m}\left(\frac{a+1}{2}\right)_{m}(a-b-c)_{m}(\gamma+1)_{m}\left(a_{1}\right)_{m} \cdots\left(a_{r}\right)_{m}(-n)_{m}(-4 x)^{m}}{m !(1+a-b)_{m}(1+a-c)_{m}(\gamma)_{m}\left(b_{1}\right)_{m} \cdots\left(b_{s}\right)_{m}}\right. \\
& \left.\times{ }_{r+2} F_{s}\left(\begin{array}{c}
a+2 m, a_{1}+m, \ldots, a_{r}+m,-n+m \\
b_{1}+m, \ldots, b_{s}+m
\end{array} \mid x\right)\right) .
\end{aligned}
$$

Proof. Using (2.6), we have

$$
\begin{aligned}
& { }_{r+6} F_{s+4}\left(\begin{array}{c}
a, b, c, a-p+1, p+1, a_{1}, \ldots, a_{r},-n \\
1+a-b, 1+a-c, p, a-p, b_{1}, \ldots, b_{s}
\end{array} \mid x\right) \\
& =\sum_{k=0}^{n} \frac{(a)_{k}(b)_{k}(c)_{k}(a-p+1)_{k}(p+1)_{k}\left(a_{1}\right)_{k} \cdots\left(a_{r}\right)_{k}(-n)_{k} x^{k}}{k !(1+a-b)_{k}(1+a-c)_{k}(p)_{k}(a-p)_{k}\left(b_{1}\right)_{k} \cdots\left(b_{s}\right)_{k}} \\
& =\sum_{k=0}^{n}\left(\frac{(a)_{k}\left(a_{1}\right)_{k} \cdots\left(a_{r}\right)_{k}(-n)_{k} x^{k}}{k !\left(b_{1}\right)_{k} \cdots\left(b_{s}\right)_{k}}\right. \\
& \left.\times{ }_{4} F_{3}\left(\begin{array}{c}
a-b-c, \gamma+1, a+k,-k \\
1+a-b, 1+a-c, \gamma
\end{array}\right)\right),
\end{aligned}
$$

where

$$
\gamma=\frac{p(a-p)(b+c-a)}{b c-p(a-p)} .
$$

We write the ${ }_{4} F_{3}$ series on the right-hand side above as a summation, switch the order of summation in the resulting expression, and then simplify to obtain (3.2). 
Remark 3.2. Formula (3.2) is an extension of [2, Eq. 4.3.1]. In fact, [2, Eq. 4.3.1] follows from (3.2) by letting $x=1$ and $p \rightarrow \infty$.

We next use Proposition 3.1 to obtain a generalization of [2, Eqs. 4.5.1 and 4.5.2]:

Proposition 3.3. We have

$$
\begin{aligned}
& { }_{7} F_{6}\left(\begin{array}{c}
a, b, c, a-p+1, p+1, q+1,-n \\
1+a-b, 1+a-c, p, a-p, q, w
\end{array} \mid 1\right) \\
& =\frac{(w-a-1)_{n}(\alpha+1)_{n}}{(w)_{n}(\alpha)_{n}} \\
& \times{ }_{7} F_{6}\left(\begin{array}{c}
1+a-w, \frac{a}{2}, \frac{a+1}{2}, a-b-c, \beta+1, \gamma+1,-n \\
1+a-b, 1+a-c, \frac{2+a-w-n}{2}, \frac{3+a-w-n}{2}, \beta, \gamma
\end{array} \mid 1\right)
\end{aligned}
$$

where

$$
\begin{gathered}
\alpha=\frac{q(1+a-w)}{a-q}, \\
\beta=\frac{q(1+a-w)+n(a-q)}{1+2 q-w+n}
\end{gathered}
$$

and

$$
\gamma=\frac{p(a-p)(b+c-a)}{b c-p(a-p)} .
$$

Proof. Use $q+1, q, w, 1$ for $a_{1}, b_{1}, b_{2}, x$, respectively, in (3.2) to obtain

$$
\begin{aligned}
& { }_{7} F_{6}\left(\begin{array}{c}
a, b, c, a-p+1, p+1, q+1,-n \\
1+a-b, 1+a-c, p, a-p, q, w
\end{array} \mid 1\right) \\
& =\sum_{m=0}^{n}\left(\frac{\left(\frac{a}{2}\right)_{m}\left(\frac{a+1}{2}\right)_{m}(a-b-c)_{m}(\gamma+1)_{m}(q+1)_{m}(-n)_{m}(-4)^{m}}{m !(1+a-b)_{m}(1+a-c)_{m}(\gamma)_{m}(q)_{m}(w)_{m}}\right. \\
& \left.\times{ }_{3} F_{2}\left(\begin{array}{c|c}
a+2 m, q+1+m,-n+m & 1
\end{array}\right)\right),
\end{aligned}
$$

where

$$
\gamma=\frac{p(a-p)(b+c-a)}{b c-p(a-p)} .
$$

Sum the ${ }_{3} F_{2}$ series on the right-hand side above according to (2.2) and simplify to obtain the result. 
We note that the ${ }_{7} F_{6}$ series on the left-hand side of (3.3) deviates from a well-poised series in two pairs of numerator and denominator parameters while the ${ }_{7} F_{6}$ series on the right-hand side of (3.3) is Saalschützian.

Letting $q \rightarrow \infty$ in (3.3), we obtain the following special case:

Corollary 3.4. We have

$$
\begin{aligned}
& { }_{6} F_{5}\left(\begin{array}{c}
a, b, c, a-p+1, p+1,-n \\
1+a-b, 1+a-c, p, a-p, w
\end{array} \mid 1\right) \\
& =\frac{(w-a)_{n}}{(w)_{n}} \\
& \times{ }_{6} F_{5}\left(\begin{array}{c}
1+a-w, \frac{a}{2}, \frac{a+1}{2}, a-b-c, \gamma+1,-n \\
1+a-b, 1+a-c, \frac{1+a-w-n}{2}, \frac{2+a-w-n}{2}, \gamma
\end{array} \mid 1\right),
\end{aligned}
$$

where

$$
\gamma=\frac{p(a-p)(b+c-a)}{b c-p(a-p)} .
$$

We remark that (3.7) is the special case $k=1$ of [9, Theorem 7.1(ii)] as well as [17, Cor. 4].

On the other hand, letting $p \rightarrow \infty$ in (3.3), we obtain the following result:

Corollary 3.5. We have

$$
\begin{aligned}
& { }_{5} F_{4}\left(\begin{array}{c}
a, b, c, q+1,-n \\
1+a-b, 1+a-c, q, w
\end{array} \mid 1\right) \\
& =\frac{(w-a-1)_{n}(\alpha+1)_{n}}{(w)_{n}(\alpha)_{n}} \\
& \times{ }_{6} F_{5}\left(\begin{array}{c}
1+a-w, \frac{a}{2}, \frac{a+1}{2}, 1+a-b-c, \beta+1,-n \\
1+a-b, 1+a-c, \frac{2+a-w-n}{2}, \frac{3+a-w-n}{2}, \beta
\end{array} \mid 1\right),
\end{aligned}
$$

where

$$
\alpha=\frac{q(1+a-w)}{a-q}
$$

and

$$
\beta=\frac{q(1+a-w)+n(a-q)}{1+2 q-w+n} .
$$


We note that Corollary 3.5 generalizes two well-known results of Whipple and Bailey given in [2, Eqs. 4.5.1 and 4.5.2]. Indeed, we have the following:

(a) Letting $q \rightarrow \infty$ in (3.9) gives [2, Eq. 4.5.1] (originally found by Whipple in $[22])$.

(b) Letting $q=a / 2$ in (3.9) gives [2, Eq. 4.5.2] (originally found by Bailey in [1]).

We next show how Proposition 3.1 along with the result in Corollary 3.5 lead to a formula that generalizes transformations of both nearly-poised and very-well-poised series to Saalschützian series.

Proposition 3.6. We have

$$
\begin{aligned}
& { }_{9} F_{8}\left(\begin{array}{c}
a, b, c, d, e, a-p+1, p+1, q+1,-n \\
1+a-b, 1+a-c, 1+a-d, 1+a-e, p, a-p, q, w
\end{array} \mid 1\right) \\
& =\frac{(w-a-1)_{n}(\alpha+1)_{n}}{(w)_{n}(\alpha)_{n}} \\
& \times \sum_{k=0}^{n}\left(\frac{(-n)_{k}\left(\frac{a}{2}\right)_{k}\left(\frac{a+1}{2}\right)_{k}(1+a-w)_{k}(1+a-d-e)_{k}(\beta+1)_{k}}{k !(1+a-d)_{k}(1+a-e)_{k}\left(\frac{2+a-w-n}{2}\right)_{k}\left(\frac{3+a-w-n}{2}\right)_{k}(\beta)_{k}}\right. \\
& \times{ }_{5} F_{4}\left(\begin{array}{c}
-k, a-b-c, d, e, \gamma+1 \\
1+a-b, 1+a-c, d+e-a-k, \gamma
\end{array} \mid \begin{array}{c}
1 \\
1+a
\end{array}\right),
\end{aligned}
$$

where

$$
\begin{gathered}
\alpha=\frac{q(1+a-w)}{a-q}, \\
\beta=\frac{q(1+a-w)+n(a-q)}{1+2 q-w+n}
\end{gathered}
$$

and

$$
\gamma=\frac{p(a-p)(b+c-a)}{b c-p(a-p)}
$$

Proof. Use $d, e, q+1$ for $a_{1}, a_{2}, a_{3}$, respectively, $1+a-d, 1+a-e, q, w$ for $b_{1}, b_{2}, b_{3}, b_{4}$, respectively, and $x=1$ in (3.2), and then apply (3.9) to write the ${ }_{5} F_{4}$ series on the right-hand side as a Saalschützian ${ }_{6} F_{5}$ series. After that reverse the order of summation and simplify. 
The formula in Proposition 3.6 is a very general one. It extends both very-well-poised identities as well as nearly-poised identities. In fact, letting $q \rightarrow a / 2$ first in (3.12) and then letting $w \rightarrow 1+a+n$ in the resulting formula yields

$$
\begin{aligned}
& { }_{9} F_{8}\left(\begin{array}{c}
a, 1+\frac{a}{2}, b, c, d, e, a-p+1, p+1,-n \\
\frac{a}{2}, 1+a-b, 1+a-c, 1+a-d, 1+a-e, p, a-p, 1+a+n
\end{array} \mid 1\right) \\
& =\frac{(1+a)_{n}(1+a-d-e)_{n}}{(1+a-d)_{n}(1+a-e)_{n}} \\
& \left.\times{ }_{5} F_{4}\left(\begin{array}{c|c}
a-b-c, d, e, \gamma+1,-n \\
1+a-b, 1+a-c, d+e-a-n, \gamma
\end{array} \mid 1\right)\right),
\end{aligned}
$$

where

$$
\gamma=\frac{p(a-p)(b+c-a)}{b c-p(a-p)}
$$

which is the very-well-poised ${ }_{9} F_{8}(1)$ to Saalschützian ${ }_{5} F_{4}(1)$ transformation found by Srivastava, Vyas and Fatawat in [15, Theorem 3.2] that generalizes the classical Whipple's transformation of a very-well-poised ${ }_{7} F_{6}(1)$ series to a Saalschützian ${ }_{4} F_{3}(1)$ series (see [19], [21] and [2, Eq. 4.3.4]). On the other

hand, letting $b \rightarrow \infty$ in (3.12) and then letting $c \rightarrow \infty$ in the resulting formula leads to (3.3), which greatly generalizes the classical nearly-poised to Saalschützian transformations found by Whipple and Bailey (see [2, Eqs. 4.5.1 and 4.5.2]).

\section{Extensions of hypergeometric transforma- tions of Saalschützian to Saalschützian se- ries}

In this section, we extend the well-known Whipple transform (see [19], 20] and [2, Eq. 7.2.1]) which involves two terminating Saalschützian ${ }_{4} F_{3}(1)$ series. We begin with the following general result:

Proposition 4.1. Let

$$
\gamma=\frac{p(c-a-1)(c-b-1)}{a b+p(c-a-b-1)}
$$


Then

$$
\begin{aligned}
& { }_{r+4} F_{s+2}\left(\begin{array}{c}
a, b, p+1, a_{1}, \ldots, a_{r},-n \\
c, p, b_{1}, \ldots, b_{s}
\end{array} \mid x\right) \\
& =\sum_{m=0}^{n}\left(\frac{(c-a-1)_{m}(c-b-1)_{m}(\gamma+1)_{m}\left(a_{1}\right)_{m} \cdots\left(a_{r}\right)_{m}(-n)_{m} x^{m}}{m !(c)_{m}(\gamma)_{m}\left(b_{1}\right)_{m} \cdots\left(b_{s}\right)_{m}}\right. \\
& \left.\times{ }_{r+2} F_{s}\left(\begin{array}{c}
1+a+b-c, a_{1}+m, \ldots, a_{r}+m,-n+m \mid x \\
b_{1}+m, \ldots, b_{s}+m
\end{array}\right)\right)
\end{aligned}
$$

Proof. Using (2.8), we have

$$
\begin{aligned}
& { }_{r+4} F_{s+2}\left(\begin{array}{c}
a, b, p+1, a_{1}, \ldots, a_{r},-n \\
c, p, b_{1}, \ldots, b_{s}
\end{array} \mid x\right) \\
& =\sum_{k=0}^{n} \frac{(a)_{k}(b)_{k}(p+1)_{k}\left(a_{1}\right)_{k} \cdots\left(a_{r}\right)_{k}(-n)_{k} x^{k}}{k !(c)_{k}(p)_{k}\left(b_{1}\right)_{k} \cdots\left(b_{s}\right)_{k}} \\
& =\sum_{k=0}^{n}\left(\frac{(1+a+b-c)_{k}\left(a_{1}\right)_{k} \cdots\left(a_{r}\right)_{k}(-n)_{k} x^{k}}{k !\left(b_{1}\right)_{k} \cdots\left(b_{s}\right)_{k}}\right. \\
& \left.\times{ }_{4} F_{3}\left(\begin{array}{c}
c-a-1, c-b-1, \gamma+1,-k \\
c, \gamma, c-a-b-k
\end{array}\right)\right),
\end{aligned}
$$

where

$$
\gamma=\frac{p(c-a-1)(c-b-1)}{a b+p(c-a-b-1)} .
$$

We write the ${ }_{4} F_{3}$ series on the right-hand side above as a summation, switch the order of summation in the resulting expression, and then simplify to obtain (4.2).

The extension of the Whipple transform is given next:

Proposition 4.2. We have

$$
\begin{aligned}
& { }_{6} F_{5}\left(\begin{array}{c}
a, b, c, p+1, q+1,-n \\
d, e, f, p, q
\end{array}\right) \\
& =\frac{(e-c-1)_{n}(f-c-1)_{n}(\alpha+1)_{n}}{(e)_{n}(f)_{n}(\alpha)_{n}} \\
& \times{ }_{6} F_{5}\left(\begin{array}{c}
d-a-1, d-b-1, c, \gamma+1, \delta+1,-n \\
d, 2+c-e-n, 2+c-f-n, \gamma, \delta
\end{array}\right.
\end{aligned}
$$


where

$$
\begin{gathered}
d+e+f-a-b-c+n=3, \\
\alpha=\frac{q(e-c-1)(f-c-1)}{(c-q)(d-a-b-1)}, \\
\gamma=\frac{p(d-a-1)(d-b-1)}{a b+p(d-a-b-1)}
\end{gathered}
$$

and

$$
\delta=\frac{q(e-c-1)(f-c-1)+n(c-q)(d-a-b-1)}{(e-c-1)(f-c-1)-(c-q)(d-a-b-1)} .
$$

Proof. Use $d, c, q+1, e, f=3+a+b+c-d-e-n, q, 1$ for $c, a_{1}, a_{2}, b_{1}, b_{2}, b_{3}, x$, respectively, in (4.2), and then sum the Saalschützian ${ }_{4} F_{3}(1)$ series on the right-hand side according to (2.4).

The relation in (4.3) involves two Saalschützian ${ }_{6} F_{5}(1)$ series in each of which series two numerator parameters exceed two denominator parameters by one. This relation is a generalization of the classical Whipple transform (see [19], 20] and [2, Eq. 7.2.1]) involving two terminating Saalschützian ${ }_{4} F_{3}(1)$ series as we show after Corollary 4.4 below.

Remark 4.3. Let

$$
\begin{aligned}
& \tilde{F}_{n}(a, b, c ; d, e, f ; p, q) \\
& =(d)_{n}(e)_{n}(f)_{n}(\alpha)_{n 6} F_{5}\left(\begin{array}{c|c}
a, b, c, p+1, q+1,-n & 1 \\
d, e, f, p, q & 1
\end{array}\right),
\end{aligned}
$$

where

$$
d+e+f-a-b-c+n=3
$$

and $\alpha$ is as given in (4.5). Then equation (4.3) implies that

$$
\begin{aligned}
& \tilde{F}_{n}(a, b, c ; d, e, f ; p, q) \\
& =(-1)^{n} \tilde{F}_{n}(d-a-1, d-b-1, c ; d, 2+c-e-n, 2+c-f-n ; \gamma, \delta),
\end{aligned}
$$

where $\gamma$ and $\delta$ are as given in (4.6) and (4.7), respectively. 
Corollary 4.4. We have

$$
\begin{aligned}
& { }_{5} F_{4}\left(\begin{array}{c}
a, b, c, p+1,-n \\
d, e, f, p
\end{array} \mid 1\right) \\
& =\frac{(e-c)_{n}(f-c)_{n}}{(e)_{n}(f)_{n}} \\
& \times_{5} F_{4}\left(\begin{array}{c}
d-a-1, d-b-1, c, \gamma+1,-n \\
d, 1+c-e-n, 1+c-f-n, \gamma
\end{array} \mid 1\right),
\end{aligned}
$$

where

$$
d+e+f-a-b-c+n=2,
$$

and

$$
\gamma=\frac{p(d-a-1)(d-b-1)}{a b+p(d-a-b-1)} .
$$

Proof. Let $q \rightarrow c$ in (4.3) and then replace $c+1$ with $c$.

The two ${ }_{5} F_{4}(1)$ series in (4.10) are both Saalschützian and in each one of them a numerator parameter exceeds a denominator parameter by one. Letting $p=b$ in (4.10) gives the Whipple transform involving two Saalschützian ${ }_{4} F_{3}(1)$ series.

Corollary 4.5. We have

$$
\begin{aligned}
& { }_{4} F_{3}\left(\begin{array}{c|c}
a, c, p+1,-n & 1 \\
d, e, p & 1
\end{array}\right) \\
& =\frac{(e-c)_{n}}{(e)_{n}} F_{3}\left(\begin{array}{c}
d-a-1, c, \gamma+1,-n \\
d, 1+c-e-n, \gamma
\end{array} \mid 1\right) \text {, }
\end{aligned}
$$

where

$$
\gamma=\frac{p(d-a-1)}{p-a} .
$$

Proof. In (4.10), fix $a, c, d, e, p$ and $n$, and let

$$
f=2+a+b+c-d-e-n
$$

depend on $b$. Let $b \rightarrow \infty$ to obtain the result. 
We note that (4.13) generalizes the classical relation involving two terminating ${ }_{3} F_{2}(1)$ series (see Sheppard [13] and Whipple [18] which follow Thomae [16]). Indeed, letting $p \rightarrow \infty$ in (4.13) gives

$$
\begin{aligned}
& { }_{3} F_{2}\left(\begin{array}{c}
a, c,-n \\
d, e
\end{array} \mid 1\right) \\
& =\frac{(e-c)_{n}}{(e)_{n}}{ }_{3} F_{2}\left(\begin{array}{c}
d-a, c,-n \\
d, 1+c-e-n
\end{array}\right.
\end{aligned}
$$

Equation (4.13) also extends the Saalschützian ${ }_{4} F_{3}(1)$ summation (2.4). In fact, (2.4) follows from (4.13) by setting $e=2+a+c-d-n$ and then summing the resulting ${ }_{3} F_{2}(1)$ series on the right-hand side according to (2.2).

\section{Extensions of hypergeometric transforma- tions of very-well-poised and nearly-poised series to very-well-poised series}

In this section, we extend the relation between two terminating very-wellpoised ${ }_{11} F_{10}(1)$ series given by Srivastava, Vyas and Fatawat in [15, Theorem 3.4] (which generalizes Bailey's ${ }_{9} F_{8}$ transformation in [2, Eq. 4.3.7]) to a relation between two terminating very-well-poised ${ }_{13} F_{12}(1)$ series. We also extend the formulas found in [2, Eqs. 4.5.3, 4.5.4 and 4.5.5]. We begin with a general formula that extends [2, Eq. 4.3.6]:

Proposition 5.1. If

$$
\lambda=2 a-b-c-d,
$$

then

$$
\begin{aligned}
& { }_{r+7} F_{s+5}\left(\begin{array}{c}
a, b, c, d, a-p+1, p+1, a_{1}, \ldots, a_{r},-n \\
1+a-b, 1+a-c, 1+a-d, p, a-p, b_{1}, \ldots, b_{s}
\end{array} \mid x\right) \\
& =\sum_{m=0}^{n}\left(\frac{(\lambda)_{m}(\lambda+b-a)_{m}(\lambda+c-a)_{m}(\lambda+d-a)_{m}\left(\frac{a}{2}\right)_{m}\left(\frac{a+1}{2}\right)_{m}}{m !\left(\frac{\lambda}{2}\right)_{m}\left(\frac{\lambda+1}{2}\right)_{m}(1+a-b)_{m}(1+a-c)_{m}(1+a-d)_{m}}\right. \\
& \times \frac{\left(\frac{\lambda}{2}-\gamma+1\right)_{m}\left(\frac{\lambda}{2}+\gamma+1\right)_{m}\left(a_{1}\right)_{m} \cdots\left(a_{r}\right)_{m}(-n)_{m} x^{m}}{\left(\frac{\lambda}{2}+\gamma\right)_{m}\left(\frac{\lambda}{2}-\gamma\right)_{m}\left(b_{1}\right)_{m} \cdots\left(b_{s}\right)_{m}} \\
& \left.\times{ }_{r+3} F_{s+1}\left(\begin{array}{c}
a+2 m, a-\lambda, a_{1}+m, \ldots, a_{r}+m,-n+m \\
1+\lambda+2 m, b_{1}+m, \ldots, b_{s}+m
\end{array} \mid x\right)\right)
\end{aligned}
$$


where

$$
\gamma^{2}=\frac{\lambda^{2}}{4}-\frac{p(a-p)(a-b-c)(a-b-d)(a-c-d)}{b c d+p(a-p)(a-b-c-d)}
$$

Proof. Using (2.12), we have

$$
\begin{aligned}
& { }_{r+7} F_{s+5}\left(\begin{array}{c}
a, b, c, d, a-p+1, p+1, a_{1}, \ldots, a_{r},-n \\
1+a-b, 1+a-c, 1+a-d, p, a-p, b_{1}, \ldots, b_{s}
\end{array} \mid x\right) \\
& =\sum_{k=0}^{n} \frac{(a)_{k}(b)_{k}(c)_{k}(d)_{k}(a-p+1)_{k}(p+1)_{k}}{k !(1+a-b)_{k}(1+a-c)_{k}(1+a-d)_{k}(p)_{k}(a-p)_{k}} \\
& \times \frac{\left(a_{1}\right)_{k} \cdots\left(a_{r}\right)_{k}(-n)_{k} x^{k}}{\left(b_{1}\right)_{k} \cdots\left(b_{s}\right)_{k}} \\
& =\sum_{k=0}^{n}\left(\frac{(a)_{k}(a-\lambda)_{k}\left(a_{1}\right)_{k} \cdots\left(a_{r}\right)_{k}(-n)_{k} x^{k}}{k !(1+\lambda)_{k}\left(b_{1}\right)_{k} \cdots\left(b_{s}\right)_{k}}\right. \\
& { }_{9} F_{8}\left(\begin{array}{c}
\lambda, 1+\frac{\lambda}{2}, \lambda+b-a, \lambda+c-a, \lambda+d-a, \\
\frac{\lambda}{2}, 1+a-b, 1+a-c, 1+a-d,
\end{array}\right. \\
& \left.\left.\begin{array}{c|c}
a+k, \frac{\lambda}{2}-\gamma+1, \frac{\lambda}{2}+\gamma+1,-k ; \\
+\lambda-a-k, \frac{\lambda}{2}+\gamma, \frac{\lambda}{2}-\gamma, 1+\lambda+k ;
\end{array}\right)\right),
\end{aligned}
$$

where

$$
\lambda=2 a-b-c-d
$$

and

$$
\gamma^{2}=\frac{\lambda^{2}}{4}-\frac{p(a-p)(a-b-c)(a-b-d)(a-c-d)}{b c d+p(a-p)(a-b-c-d)} .
$$

We write the ${ }_{9} F_{8}$ series on the right-hand side above as a summation, switch the order of summation in the resulting expression, and then simplify to obtain (5.2).

Remark 5.2. Formula (5.2) is an extension of [2, Eq. 4.3.6]. In fact, [2, Eq. 4.3.6] follows from (5.2) by letting $x=1$ and $p=d$.

We now obtain the generalization of Srivastava, Vyas and Fatawat's ${ }_{11} F_{10}$ transformation given in [15, Theorem 3.4]:

Proposition 5.3. Suppose

$$
3 a=b+c+d+e+f+g-n .
$$


Then

$$
\begin{aligned}
& \quad a, 1+\frac{a}{2}, b, c, d, e, f, \\
& { }_{13} F_{12}\left(\begin{array}{c}
a \\
\frac{a}{2}, 1+a-b, 1+a-c, 1+a-d, 1+a-e, 1+a-f,
\end{array}\right. \\
& g, a-p+1, p+1, a-q+1, q+1,-n \mid 1) \\
& 1+a-g, p, a-p, q, a-q, 1+a+n \mid) \\
& =\frac{(1+a)_{n}(1+\lambda-e)_{n}(1+\lambda-f)_{n}(1+\lambda-g)_{n}}{(1+\lambda)_{n}(1+a-e)_{n}(1+a-f)_{n}(1+a-g)_{n}} \\
& \times \frac{\left(\frac{\mu}{2}-\delta+1\right)_{n}\left(\frac{\mu}{2}+\delta+1\right)_{n}}{\left(\frac{\mu}{2}+\delta\right)_{n}\left(\frac{\mu}{2}-\delta\right)_{n}} \quad \lambda, 1+\frac{\lambda}{2}, \lambda+b-a, \lambda+c-a, \lambda+d-a, e, f, \\
& \times{ }_{13} F_{12}\left(\frac{\lambda}{2}, 1+a-b, 1+a-c, 1+a-d, 1+\lambda-e, 1+\lambda-f,\right. \\
& g, \frac{\lambda}{2}-\gamma+1, \frac{\lambda}{2}+\gamma+1, \frac{\lambda}{2}-\epsilon+1, \frac{\lambda}{2}+\epsilon+1,-n \mid \\
& 1+\lambda-g, \frac{\lambda}{2}+\gamma, \frac{\lambda}{2}-\gamma, \frac{\lambda}{2}+\epsilon, \frac{\lambda}{2}-\epsilon, 1+\lambda+n \mid
\end{aligned}
$$

where

$$
\begin{gathered}
\lambda=2 a-b-c-d, \\
\mu=2 a-e-f-g, \\
\gamma^{2}=\frac{\lambda^{2}}{4}-\frac{p(a-p)(a-b-c)(a-b-d)(a-c-d)}{b c d+p(a-p)(a-b-c-d)} \\
\delta^{2}=\frac{\mu^{2}}{4}-\frac{q(a-q)(a-e-f)(a-e-g)(a-f-g)}{e f g+q(a-q)(a-e-f-g)}
\end{gathered}
$$

and

$$
\begin{aligned}
& \epsilon^{2}=\frac{\lambda^{2}}{4} \\
& -\frac{\left[\begin{array}{c}
q(a-q)(a-e-f)(a-e-g)(a-f-g) \\
+n(\mu+n)(e f g+q(a-q)(a-e-f-g))
\end{array}\right]}{\left[\begin{array}{c}
(a-e-f)(a-e-g)(a-f-g) \\
-(\mu+n)(e f+e g+f g+a(a-e-f-g)-q(a-q))
\end{array}\right]} .
\end{aligned}
$$


Proof. Use $1+\frac{a}{2}, e, f, g, a-q+1, q+1$ for $a_{1}, a_{2}, a_{3}, a_{4}, a_{5}, a_{6}$, respectively, $\frac{a}{2}, 1+a-e, 1+a-f, 1+a-g, q, a-q, 1+a+n$ for $b_{1}, b_{2}, b_{3}, b_{4}, b_{5}, b_{6}, b_{7}$, respectively, and $x=1$ in (5.2), and then apply (2.10) to sum the ${ }_{9} F_{8}(1)$ series on the right-hand side. The result follows after some simplification.

Equation (5.5) above involves two terminating very-well-poised ${ }_{13} F_{12}(1)$ series. It generalizes the result of Srivastava, Vyas and Fatawat [15, Theorem 3.4] between two terminating very-well-poised ${ }_{11} F_{10}(1)$ series, which in turn is a generalization of Bailey's ${ }_{9} F_{8}$ transformation (see [2, Eq. 4.3.7]). Indeed, [15, Theorem 3.4] follows from our result (5.5) upon setting $q=e$.

We next extend the formulas found in [2, Eqs. 4.5.3, 4.5.4 and 4.5.5]. First, we obtain an even more general result:

Proposition 5.4. We have

$$
\begin{aligned}
& { }_{8} F_{7}\left(\begin{array}{c}
a, b, c, d, a-p+1, p+1, q+1,-n \\
1+a-b, 1+a-c, 1+a-d, p, a-p, q, w
\end{array} \mid 1\right) \\
& =\frac{(2 \lambda-a)_{n}(\lambda-a)_{n}(\alpha+1)_{n}}{(1+\lambda)_{n}(2 \lambda-2 a)_{n}(\alpha)_{n}} \\
& \times{ }_{13} F_{12}\left(\begin{array}{c}
\lambda, 1+\frac{\lambda}{2}, \frac{a}{2}, \frac{a+1}{2}, \lambda+b-a, \lambda+c-a, \lambda+d-a \\
\frac{\lambda}{2}, \frac{2+2 \lambda-a}{2}, \frac{1+2 \lambda-a}{2}, 1+a-b, 1+a-c, 1+a-d \\
1+a-w, \frac{\lambda}{2}-\gamma+1, \frac{\lambda}{2}+\gamma+1, \frac{\lambda}{2}-\delta+1, \frac{\lambda}{2}+\delta+1,-n \mid \\
\lambda+w-a, \frac{\lambda}{2}+\gamma, \frac{\lambda}{2}-\gamma, \frac{\lambda}{2}+\delta, \frac{\lambda}{2}-\delta, 1+\lambda+n
\end{array}\right)
\end{aligned}
$$

where

$$
\begin{gathered}
\lambda=2 a-b-c-d, \\
w=1+2 a-2 \lambda-n, \\
\alpha=\frac{q(2 \lambda-a)}{2 q-a} \\
\gamma^{2}=\frac{\lambda^{2}}{4}-\frac{p(a-p)(a-b-c)(a-b-d)(a-c-d)}{b c d+p(a-p)(a-b-c-d)}
\end{gathered}
$$

and

$$
\delta^{2}=\frac{\lambda^{2}}{4}-\frac{q(2 \lambda-a)+n(2 q-a)}{2} .
$$


Proof. Use $q+1, q, w, 1$ for $a_{1}, b_{1}, b_{2}, x$, respectively, in (5.2) (where $w$ is as given in (5.13) $)$ and then apply (2.4) to sum the Saalschützian ${ }_{4} F_{3}(1)$ series on the right-hand side. The final result follows after some simplification.

We note that the terminating ${ }_{8} F_{7}(1)$ series on the left-hand side of (5.11) is Saalschützian (i.e. with parametric excess $\omega=1$ ) and deviates from a well-poised series in two pairs of numerator and denominator parameters. The terminating ${ }_{13} F_{12}(1)$ series on the right-hand side of (5.11) is very-wellpoised.

The special case of Proposition 5.4 given in the next corollary is a direct extension of the results found in [2, Eqs. 4.5.3, 4.5.4 and 4.5.5]:

Corollary 5.5. We have

$$
\begin{aligned}
& { }_{6} F_{5}\left(\begin{array}{c}
a, b, c, d, q+1,-n \\
1+a-b, 1+a-c, 1+a-d, q, w
\end{array} \mid 1\right) \\
& =\frac{(2 \lambda-a)_{n}(\lambda-a)_{n}(\alpha+1)_{n}}{(1+\lambda)_{n}(2 \lambda-2 a)_{n}(\alpha)_{n}} \\
& \times{ }_{11} F_{10}\left(\begin{array}{c}
\lambda, 1+\frac{\lambda}{2}, \frac{a}{2}, \frac{a+1}{2}, \lambda+b-a, \lambda+c-a, \lambda+d-a, \\
\frac{\lambda}{2}, \frac{2+2 \lambda-a}{2}, \frac{1+2 \lambda-a}{2}, 1+a-b, 1+a-c, 1+a-d, \\
1+a-w, \frac{\lambda}{2}-\delta+1, \frac{\lambda}{2}+\delta+1,-n \mid \\
\lambda+w-a, \frac{\lambda}{2}+\delta, \frac{\lambda}{2}-\delta, 1+\lambda+n
\end{array}\right),
\end{aligned}
$$

where

$$
\begin{gathered}
\lambda=1+2 a-b-c-d, \\
w=1+2 a-2 \lambda-n, \\
\alpha=\frac{q(2 \lambda-a)}{2 q-a}
\end{gathered}
$$

and

$$
\delta^{2}=\frac{\lambda^{2}}{4}-\frac{q(2 \lambda-a)+n(2 q-a)}{2} .
$$

Proof. Let $p=b$ in (5.11) and then replace $b+1$ with $b$. 
Equation (5.17) above expresses a certain terminating Saalschützian (i.e. with parametric excess $\omega=1){ }_{6} F_{5}(1)$ series that deviates from a well-poised series in two pairs of numerator and denominator parameters in terms of a terminating very-well-poised ${ }_{9} F_{8}(1)$ series. This equation is a direct generalization of the classical results found by Bailey in [1, Eqs. 8.1, 8.2 and 8.3] and reproduced in [2, Eqs. 4.5.3, 4.5.4 and 4.5.5] that transform terminating nearly-poised ${ }_{5} F_{4}(1)$ series with parametric excesses $\omega=1$ and $\omega=2$ and a terminating very-well-poised ${ }_{6} F_{5}(1)$ series with parametric excess $\omega=1$ in terms of terminating very-well-poised ${ }_{9} F_{8}(1)$ series. Indeed, we have the following:

(a) Letting $q \rightarrow-n$ in (5.17) gives [2, Eq. 4.5.3].

(b) Letting $q \rightarrow \frac{a}{2}$ in (5.17) gives [2, Eq. 4.5.4].

(c) Letting $q \rightarrow \infty$ in (5.17) gives [2, Eq. 4.5.5].

\section{Extensions of classical quadratic transfor- mations}

In this last section, we show how by taking certain limits of the relations in Section 3, we can obtain generalizations of some classical quadratic transformations of hypergeometric functions. Two of the classical quadratic transformations are the following:

$$
\begin{aligned}
& { }_{3} F_{2}\left(\begin{array}{c}
a, b, c \\
1+a-b, 1+a-c
\end{array} \mid x\right) \\
& =(1-x)^{-a}{ }_{3} F_{2}\left(\begin{array}{c|c}
\frac{a}{2}, \frac{a+1}{2}, 1+a-b-c \\
1+a-b, 1+a-c
\end{array} \mid-\frac{4 x}{(1-x)^{2}}\right)
\end{aligned}
$$

and

$$
\begin{aligned}
& { }_{4} F_{3}\left(\begin{array}{c}
a, 1+\frac{a}{2}, b, c \\
\frac{a}{2}, 1+a-b, 1+a-c \mid
\end{array}\right) \\
& =(1+x)(1-x)^{-a-1}{ }_{3} F_{2}\left(\begin{array}{c}
\frac{a+1}{2}, \frac{a+2}{2}, 1+a-b-c \mid \\
1+a-b, 1+a-c
\end{array} \mid-\frac{4 x}{(1-x)^{2}}\right) .
\end{aligned}
$$


The transformation (6.1) is due to Whipple [22] and (6.2) is due to Bailey [1. The transformation (6.2) is sometimes referred to as the companion transformation of (6.1) (see [6, Section 4], for example).

In this section, we shall obtain quadratic transformations that extend both (6.1) and its companion (6.2) in terms of single transformations (see (6.3) and (6.8) below). We begin with our most general extension which follows as a consequence of Proposition 3.3 from Section 3.

Proposition 6.1. The following quadratic transformation holds:

$$
\begin{aligned}
& { }_{6} F_{5}\left(\begin{array}{c}
a, b, c, a-p+1, p+1, q+1 \\
1+a-b, 1+a-c, p, a-p, q
\end{array} \mid x\right) \\
& =\left(1+\left(\frac{a-q}{q}\right) x\right)(1-x)^{-a-1} \\
& \times_{5} F_{4}\left(\begin{array}{c}
\frac{a}{2}, \frac{a+1}{2}, a-b-c, \gamma+1, \delta+1 \\
1+a-b, 1+a-c, \gamma, \delta
\end{array} \mid-\frac{4 x}{(1-x)^{2}}\right),
\end{aligned}
$$

where

$$
\gamma=\frac{p(a-p)(b+c-a)}{b c-p(a-p)}
$$

and

$$
\delta=\frac{q+(a-q) x}{1+x} .
$$

Proof. Let $w=-n / x$ in (3.3) and then let $n \rightarrow \infty$.

The quadratic transformation (6.3) above is a very general one. It extends both (6.1) and (6.2) as we show after Corollary 6.3 below. Furthermore, the presence of the variable $x$ as part of $\delta$ that appears in the numerator and denominator parameters in the ${ }_{5} F_{4}$ series on the right-hand side of (6.3) seems to be a new feature and distinguishes this transformation from most other known transformations.

Two interesting quadratic transformations that follow as special cases of (6.3) are derived below. 
Corollary 6.2. The following quadratic transformation holds:

$$
\begin{aligned}
& { }_{5} F_{4}\left(\begin{array}{c}
a, b, c, a-p+1, p+1 \\
1+a-b, 1+a-c, p, a-p
\end{array} \mid x\right) \\
& =(1-x)^{-a} \\
& \times{ }_{4} F_{3}\left(\begin{array}{c}
\frac{a}{2}, \frac{a+1}{2}, a-b-c, \gamma+1 \\
1+a-b, 1+a-c, \gamma
\end{array} \mid-\frac{4 x}{(1-x)^{2}}\right),
\end{aligned}
$$

where

$$
\gamma=\frac{p(a-p)(b+c-a)}{b c-p(a-p)} .
$$

Proof. Let $q \rightarrow \infty$ in (6.3).

We note that the transformation (6.6) is the same as the special case $k=1$ in [9, Theorem 3.4].

Corollary 6.3. The following quadratic transformation holds:

$$
\begin{aligned}
& { }_{4} F_{3}\left(\begin{array}{c}
a, b, c, q+1 \\
1+a-b, 1+a-c, q
\end{array} \mid x\right) \\
& =\left(1+\left(\frac{a-q}{q}\right) x\right)(1-x)^{-a-1} \\
& \times{ }_{4} F_{3}\left(\begin{array}{c|c}
\frac{a}{2}, \frac{a+1}{2}, 1+a-b-c, \delta+1 \\
1+a-b, 1+a-c, \delta
\end{array} \mid-\frac{4 x}{(1-x)^{2}}\right),
\end{aligned}
$$

where

$$
\delta=\frac{q+(a-q) x}{1+x} .
$$

Proof. Let $p \rightarrow \infty$ in (6.3).

The quadratic transformation (6.8) above is the direct extension of (6.1) and its companion (6.2). In fact, Whipple's transformation (6.1) follows from (6.8) by letting $q \rightarrow \infty$, and Bailey's companion transformation (6.2) follows from (6.8) by letting $q \rightarrow \frac{a}{2}$. 
Corollary 6.4. We have, if $q \neq a / 2$,

$$
\begin{aligned}
& { }_{6} F_{5}\left(\begin{array}{c}
a, b, c, a-p+1, p+1, q+1 \\
1+a-b, 1+a-c, p, a-p, q
\end{array} \mid-1\right) \\
& =\frac{(2 q-a) 2^{-a-1}}{q} \\
& \times{ }_{4} F_{3}\left(\begin{array}{c}
\frac{a}{2}, \frac{a+1}{2}, a-b-c, \gamma+1 \\
1+a-b, 1+a-c, \gamma
\end{array}\right),
\end{aligned}
$$

where

$$
\gamma=\frac{p(a-p)(b+c-a)}{b c-p(a-p)}
$$

Proof. Let $x \rightarrow-1$ in (6.3).

The formula in (6.10) above is a generalization of Whipple's formula (see [19, 22])

$$
\begin{aligned}
& { }_{3} F_{2}\left(\begin{array}{c|c}
a, b, c \\
1+a-b, 1+a-c
\end{array} \mid-1\right) \\
& =2^{-a}{ }_{3} F_{2}\left(\begin{array}{c|c}
\frac{a}{2}, \frac{a+1}{2}, 1+a-b-c \\
1+a-b, 1+a-c
\end{array}\right) .
\end{aligned}
$$

In fact, (6.12) follows from (6.10) by letting first $q \rightarrow \infty$ and then letting $p \rightarrow \infty$ in the resulting equation.

\section{References}

[1] W.N. Bailey, Some identities involving generalized hypergemetric series, Proc. London Math. Soc. (2), 29 (1929), 503-516.

[2] W.N. Bailey, Generalized Hypergeometric Series, Cambridge University Press, Cambridge, 1935.

[3] W.N. Bailey, Some identities in combinatory analysis, Proc. London Math. Soc. (2) 49, (1947), 421-425. 
[4] W.N. Bailey, Identities of the Rogers-Ramanujan type, Proc. London Math. Soc. (2) 50, (1948), 1-10.

[5] J. Dougall, On Vandermonde's theorem and some more general expansions, Proc. Edinburgh Math. Soc., 25 (1907), 114-132.

[6] I. Gessel, D. Stanton, Strange evaluations of hypergeometric series, SIAM J. Math. Anal. 13 (1982), no. 2, 295-308.

[7] G.H. Hardy, A chapter from Ramanujan's note-book, Proc. Camb. Phil. Soc., 21 (1923), 492-503.

[8] P.W. Karlsson, Hypergeometric functions with integral parameter differences, J. Math. Phys. 12 (1971), 270-271.

[9] R.S. Maier, Extensions of the classical transformations of the hypergeometric function ${ }_{3} F_{2}$, Adv. in Appl. Math. 105 (2019), 25-47.

[10] A.R. Miller, A summation formula for Clausen's series ${ }_{3} F_{2}(1)$ with an application to Goursat's function ${ }_{2} F_{2}(1)$, J. Phys. A 38 (2005), no. 16, 3541-3545.

[11] A.P. Prudnikov, Yu.A. Brychkov, O.I. Marichev, Integrals and Series, Vol. 3, More Special Functions, Translated from the Russian by G.G. Gould, Gordon and Breach Science Publishers, New York, 1990.

[12] M. Rakha, A. Rathie, Extensions of Euler type II transformation and Saalschütz's theorem, Bull. Korean Math. Soc. 48 (2011), no. 1, 151156.

[13] W.F. Sheppard, Summation of the coefficients of some terminating hypergeometric series, Proc. London Math. Soc. (2), 10 (1912), 469-478.

[14] L.J. Slater, Generalized Hypergeometric Functions, Cambridge University Press, Cambridge, 1966.

[15] H.M. Srivastava, Y. Vyas, K. Fatawat, Extensions of the classical theorems for very well-poised hypergeometric functions, Rev. R. Acad. Cienc. Exactas Fis. Nat. Ser. A Mat. RACSAM 113 (2019), no. 2, 367-397. 
[16] J. Thomae, Ueber die Funktionen welche durch Reihen der Form dargestellt werden: $1+\frac{p p^{\prime} p^{\prime \prime}}{1 q^{\prime} q^{\prime \prime}}+\cdots$, J. Reine Angew. Math. 87 (1879), 26-73.

[17] X. Wang, A. Rathie, Extension of a quadratic transformation due to Whipple with an application, Adv. Difference Equ. 2013, 2013:157, 8pp.

[18] F.J.W. Whipple, A group of generalized hypergeometric series: relations between 120 allied series of the type $F[a, b, c ; d, e]$, Proc. London Math. Soc. (2), 24 (1926), 247-263.

[19] F.J.W. Whipple, On well-poised series, generalized hypergeometric series having parameters in pairs, each pair with the same sum, Proc. London Math Soc. (2), 24 (1926), 247-263.

[20] F.J.W. Whipple, Well-poised and other generalized hypergeometric series, Proc. London Math. Soc. (2), 25 (1926), 525-544.

[21] F.J.W. Whipple, A fundamental relation between generalized hypergeometric series, Journal London Math. Soc., 1 (1926), 138-145.

[22] F.J.W. Whipple, Some transformations of generalized hypergeometric series, Proc. London Math. Soc. (2), 26 (1927), 257-272. 